\title{
New Shape Grease Pocket for Traction Motors to Facilitate Base Oil Supply
}

\author{
Sumiko HIBINO \\ Assistant Senior Researcher, \\ Tetsuya HOSOYA \\ Lubricating Materials Laboratory, Materials Technology Division \\ Yasutomo SONE \\ Senior Researcher, \\ Lubricating Materials Laboratory, Materials Technology Division \\ Kazuo NAKAMURA \\ Senior Researcher, \\ Masaharu SUZUKI \\ Former Senior Researcher, \\ Lubricating Materials Laboratory, Materials Technology Division
}

The cycle of induction motor disassembly inspections depends on the durability of the grease, which is the least durable of all the bearing parts and materials used at present. It is therefore highly desirable to extend grease service life. To meet such requirements, a number of studies were made into grease pocket structures, which concluded that an improvement in structure was essential as grease pocket shapes have a direct relationship on grease lubrication effectiveness. A laboratory test under static conditions was performed with small grease pocket models of different shapes to formulate a grease pocket design guideline.

Keywords: grease, grease pocket, rolling bearing, tracer, tribology, traction motor

\section{Introduction}

Induction traction motors have recently been replacing direct current (DC) motors on railway rolling stock. Commutator and brush maintenance, whose periodicities are shorter than other parts and materials, is no longer required and in turn has made the disassembly inspection cycle to be determined by the durability of the bearings. Lubricating grease is the least durable of the materials used for bearings; it is therefore expected to improve grease durability to extend the maintenance cycle and result in cost-savings.

On the other hand, induction motor bearing covers have been provided with hollows (open spaces) called grease pockets to retain more grease around the bearings.

During bearing system disassembly and inspection in accordance with scheduled maintenance intervals, some amount of grease packed in grease pockets will have deteriorated to loose the capacity of lubrication, but the rest is left undamaged, so, not all grease packed around the bearing might have been effectively used.

By the way, among three types of induction motors in use which have hollows of typical different shapes in the outer cover, an investigation was made into the behavior of the grease base oil as it circulated under rotational conditions in motors installed on vehicles. As a result, base oil behavior can be improved by altering the shape of the grease pocket.

A study was made into those grease pocket characteristics that affect the behavior of the oil component in grease to find the optimal grease pocket shape from the point of view of the base oil supplied from the grease contained in the pocket.

\section{Induction motor bearings structure}

Figure 1 exemplifies a typical driving device structure. An induction motor is equipped with a cylindrical roller bearing on the gear unit side and a deep groove ball bearing on the opposite side, which act as support bearings of an internal rotor. Grease is enclosed to lubricate them.

Figure 2 shows a sectional view of the bearings. Both are fitted with covers on either side (inner and outer covers) and each has a grease pocket (GP). There are several different types of outer cover hollow shape used for the types of motors installed on vehicles. The differences in shape, like the depth or the volume of grease they contain may affect the behavior of the grease ${ }^{1)}$, however, how they affect it is not known.

Figure 3 shows the typical different shapes of the

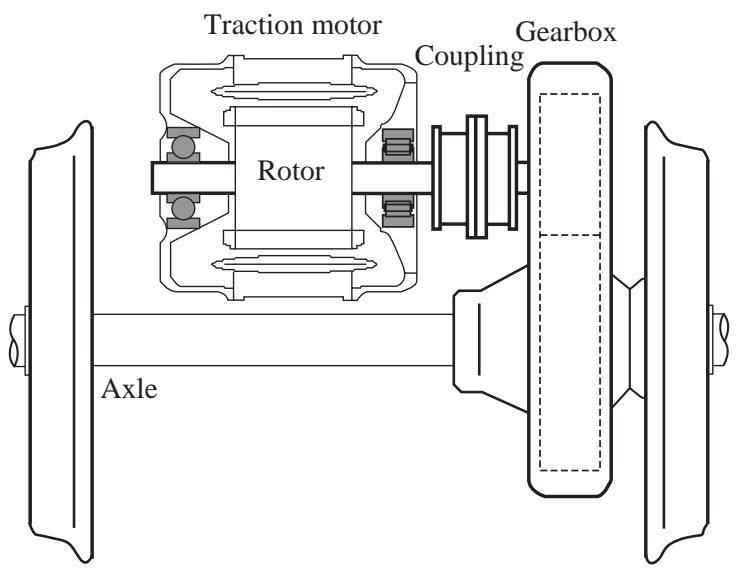

Fig. 1 Typical driving device structure 

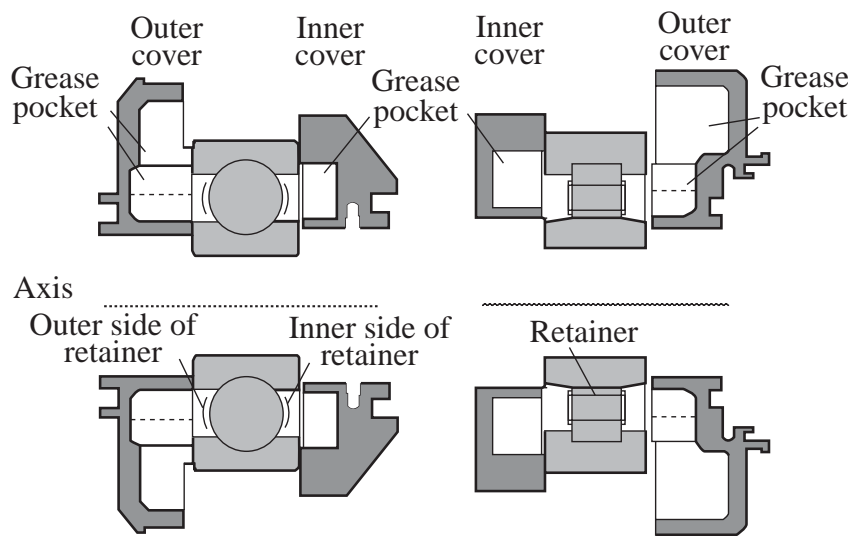

Fig. 2 Sectional view of bearings

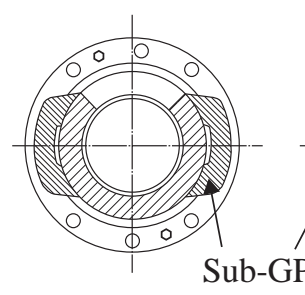

(a) MT204

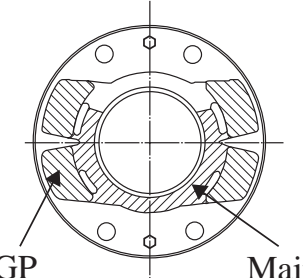

Main-GP

(Annular)

Fig. 3 Typical shapes of outer cover GPs

grease pockets in outer covers. The hatched areas contain grease. The first (Fig. 3a) is equipped with outer grease pockets (sub-GPs) on either side of an annular grease pocket (main-GP) for an MT204 motor. The second (Fig. 3b) is also equipped with sub-GPs, but divided into four sections for an MT205 motor. The last (Fig. 3c) is equipped with a main-GP only for an MT68A motor. Each type is fitted with an inner cover with a main-GP only.

\section{Test method}

\subsection{Grease test specimens}

Grease is composed of base oil as a liquid component and a thickener, which serves to retain the base oil; the properties of grease vary depending on its component combinations or manufacturing methods. During the studies into the application of the lithium-complex grease currently used for vehicle motors, it was found that the thickener component hardly moved from an original location when the grease was initially enclosed ${ }^{1)}$. The authors adopted oil-soluble blue and red pigments as oil tracers, and prepared two test greases by mixing each of them in the current grease.

\subsection{Analytical method}

After the tests, grease from each specimen was sampled to analyze the concentration of the two oil tracers. The characteristic absorption of the oil tracer in the visible absorption spectra was as shown in Fig. 4, measured with ultraviolet and visible spectrophotom-

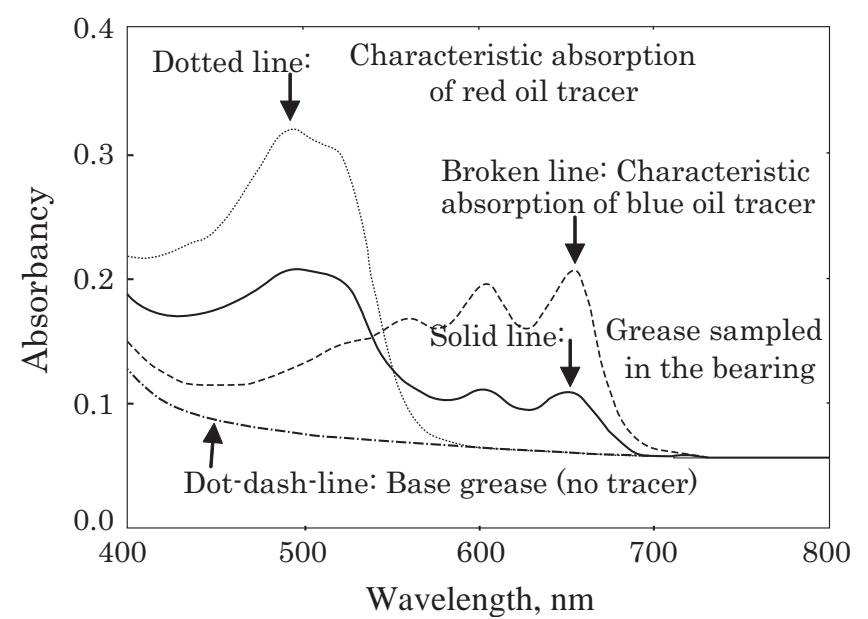

Fig. 4 Visible absorption spectra of grease sampled in the bearing

eters that estimated the volume of oil moved based on the intensity of characteristic absorption of the sampled grease (solid line).

Upon measurement, the grease was directly measured instead of being separated into base oil and thickener components. Since the measured value depends upon the oil ratio of the sampled grease, which may be varied by heating and agitation during the tests, the correct ratio of the grease sampled from representative places was accurately measured by the Soxhlet method to compensate for tracer content.

\section{Grease pocket shape}

\subsection{Depth of annular grease pocket}

In general, it is advantageous to enclose as much volume of grease as possible for lubrication purposes; however, it is not certain how the enclosed grease would be properly utilized where the hollow of a main-GP deepens.

The following tests were accordingly performed to examine the effect of deepening a GP.

Based on the MT68A traction motor currently in service, which is equipped with a main-GP only, a small working model of a GP with double the depth was prepared. Grease with a red oil tracer was enclosed in a portion to a standard depth of $6 \mathrm{~mm}$, and blue oil tracer enclosed in a deepened portion as shown in Fig. 5; a rotational test was performed using a small 6206 ball bearing for a duration of 100 hours. The test temperature was not controlled during the test.

The blue oil tracer was detected in the grease sampled from the bearings after the test, as shown with a solid line in Fig. 6. The inclined line indicating a variation of blue oil tracer concentration is almost constant to the depth indication shown in Fig. 7; oil circulation was detected in both deep and shallow portions of the GP. From the above, it was verified that grease base oil enclosed in a deeper than standard portion moved to the bearings. Therefore, the grease enclosed in deeper GPs would be properly consumed. 


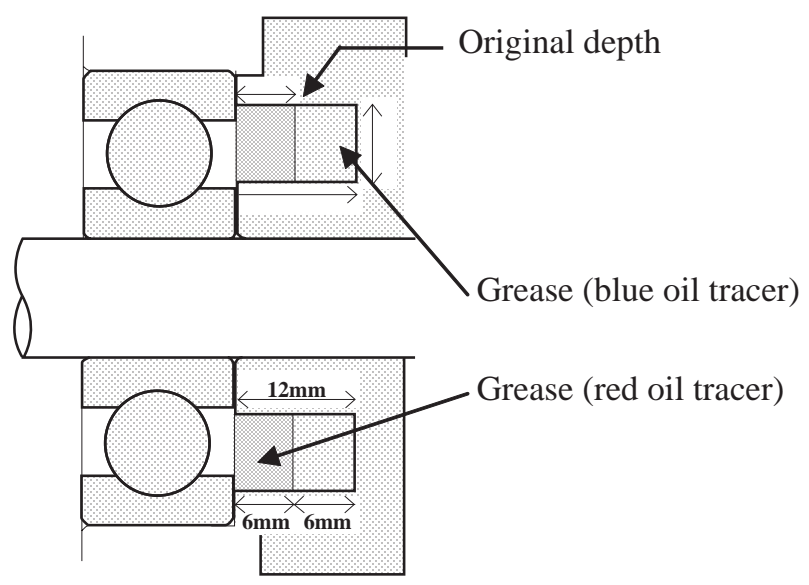

Fig. 5 First distribution of grease

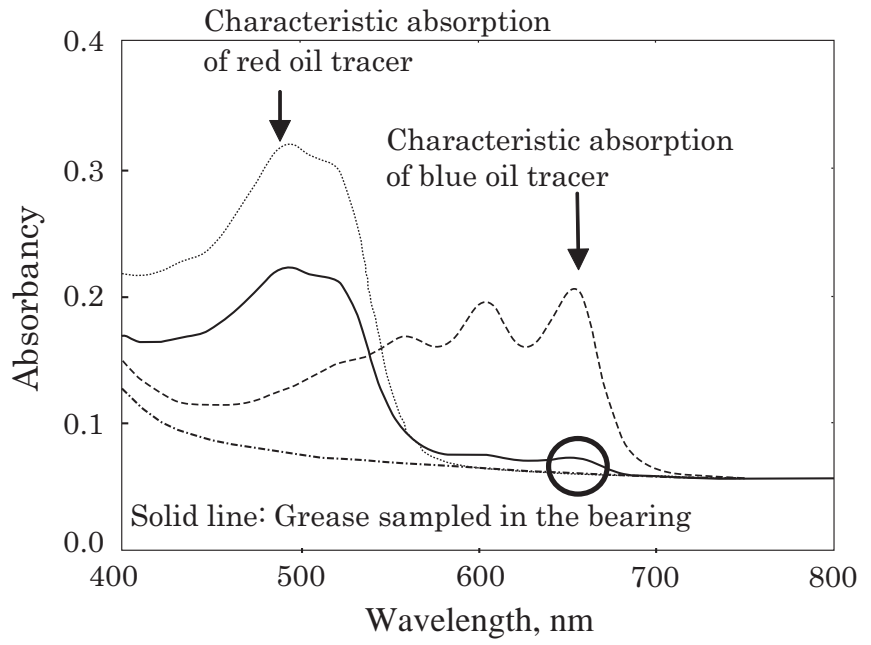

Fig. 6 Detection of deeper-packed blue oil tracer

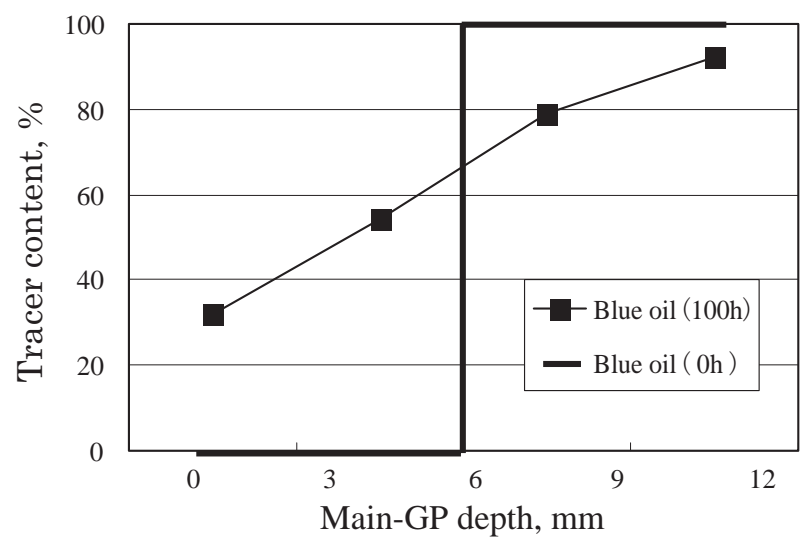

Fig. 7 Tracer content versus main-GP depth

\subsection{A comparison of sub-GP shapes}

The following tests were performed to verify the relationship between sub-GP shape and the behavior of the base oil component.

Six modified small-size sub-GP models were designed and manufactured while the main-GPs remained unchanged, as shown in Fig. 8. The models were designed based on the shape of the GP used in the existing MT204 traction motor, and the sizes adapted to 6206 ball bear-

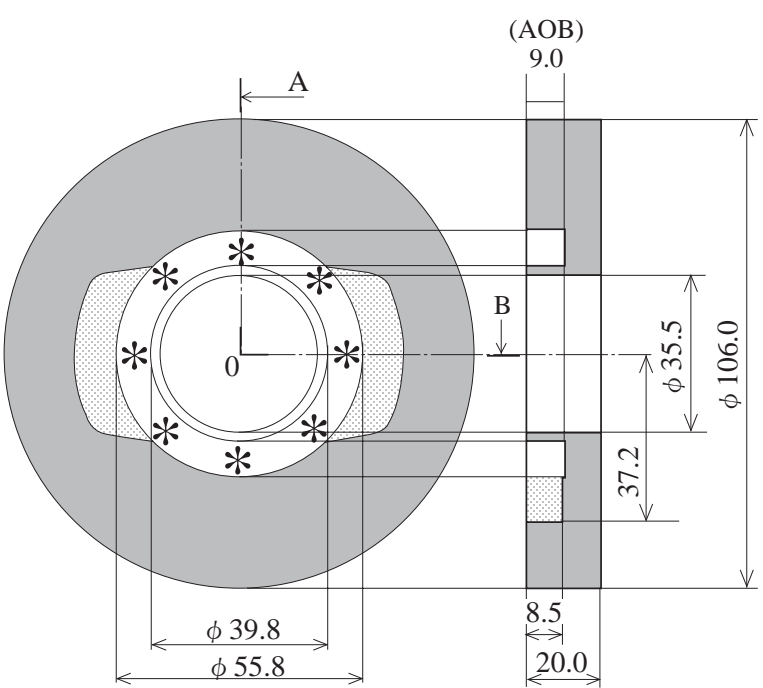

Base model (* : Grease analysis point)
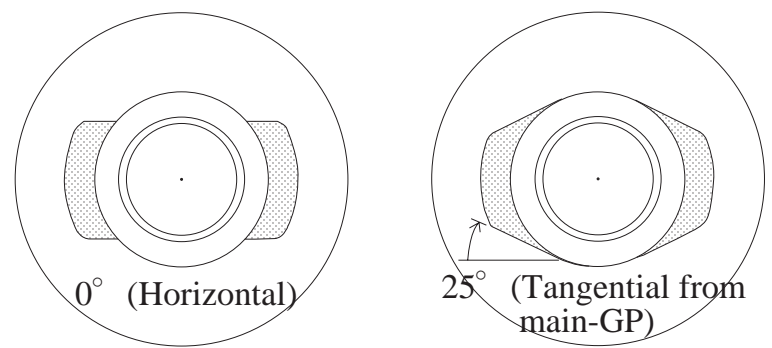

(A) Contact angle
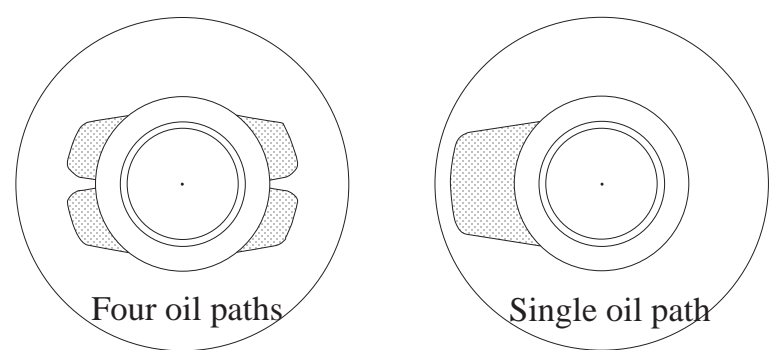

(B) Number of sub-GPs
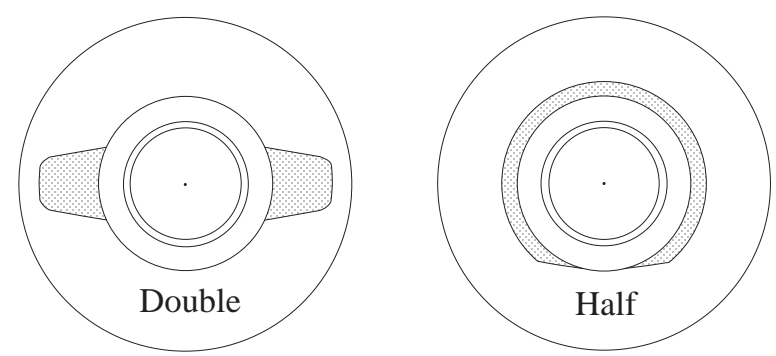

(C) Sub-GP width

Fig. 8 Model variations with different GP shapes ings.

Under the condition of constant sub-GP volume (shaded area), the contact angle of the sub-GPs to the main-GP (A), the number of sub-GPs (B) and the width of the sub-GPs relative to the diameter $(\mathrm{C})$ were respectively modified. Models (A) and (B) are objects to investigate the effect of gravity on the base oil separated from 
the grease, the models on the right side are assumed to have superior shapes.

Testing on these models was carried out at a temperature of 100 degrees Celsius for 100 hours: the subGPs were filled with grease containing a blue oil tracer, whereas the main-GPs had current grease with no tracer.

Following the test, tracer concentration was analyzed at eight points in the main-GP, as indicated by an asterisk in Fig. 8, and the results shown in Fig. 9 achieved.

(A) Where the sub-GP to main-GP contact angle was varied, there was hardly any change in concentration observed at any point in the main-GP.

(B) Where the number of sub-GPs was varied, no significant change was observed in concentration distribution throughout the main-GP.

(C) Where sub-GP width to diameter was varied, a significant change was observed in concentration distribution. Namely, a remarkable degree of
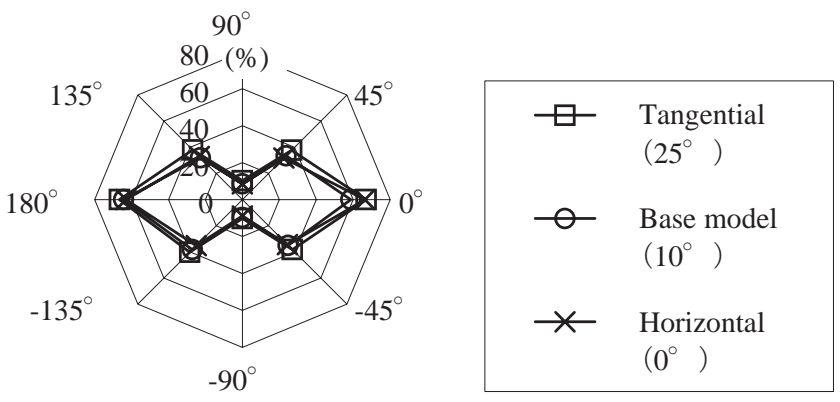

(A) Contact angle
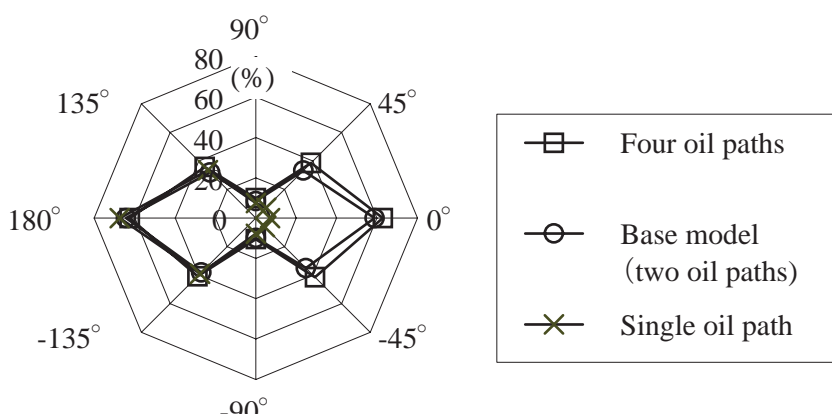

(B) Number of sub-GPs
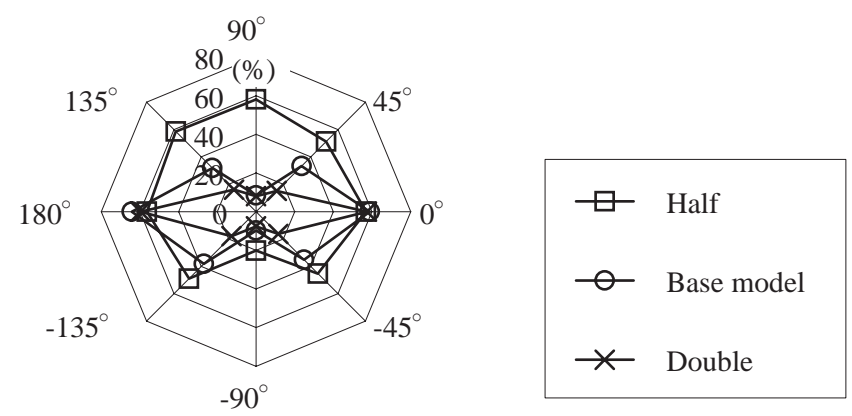

(C) Sub-GP width

Fig. 9 Oil tracer concentration in main-GP (Initial concentration of tracer : 100\%) change in concentration was observed in a halfwidth sub-GP model, which has a large contact area with the main-GP. In the case of a doublewidth sub-GP model, a lesser degree of concentration was observed except at the two points closest to the sub-GP, i.e. (0 degree and 180 degrees).

From these results, it was found that grease base oil actively circulates between main- and sub-GPs immediately after the grease is packed as shown in Fig. 9, and the behavior of the base oil depends largely on the contact area between main- and sub-GPs. The effect of gravity on base oil behavior was less than that of the contact area.

In a grease pocket designed conventionally, main-GPs are arranged to be in contact with bearing openings; subGPs are added to retain as much grease around the bearings as possible.

Since main-GPs are installed close to bearings, separated grease base oil can easily reach the bearings to provide lubrication. On the other hand, base oil from sub-GPs should pass through the main-GP to reach the bearings; therefore sub-GPs are not adequately positioned to utilize the grease oil.

According to the conventional concept, the base oil separated from the grease enclosed in sub-GPs should flow into the main-GP by gravity and then flow into the bearings. The contact angle with the sub-GPs or the differences between the bottom levels of the GPs to the bearings have been thoroughly considered.

Nevertheless, the effect due to continuity (a constant flow of grease) was larger than the difference in levels in terms of gravity between sub- and main-GPs according to the above result.

In view of the above study, it is recommended that as large a contact area as possible be provided between main- and sub-GPs where conditions permit.

\section{Conclusions}

As a result of this study, the following aspects should be taken into account in the design of grease pockets.

(1) In increasing the volume of grease, attention should first be given to deepening the main-GP or, in cases where this is difficult, the sub-GPs should be rearranged.

(2) In installing sub-GPs, the contact area with the mainGP should be as large as possible instead of merely considering the difference in levels to the bearing.

Provided that grease pockets are designed in such a manner, it is highly likely that bearings can be more durable by extending the service life of the grease.

\section{References}

1) Hibino, Hosoya, Sone, Nakamura, and Suzuki, "Shape of grease pocket to facilitate base oil migration," RTRI Report, Vol. 15, No. 7, pp. 29-34, 2001 (in Japanese) 